\title{
Study of RF Reliability of GaN HEMTs Using Low-Frequency Noise Spectroscopy
}

\author{
Hemant P. Rao and Gijs Bosman, Senior Member, IEEE
}

\begin{abstract}
The results of continuous wave short-term RF stress applied at $3 \mathrm{GHz}$ on GaN high electron mobility transistors on silicon substrate are presented. The degradation of the device characteristics for RF overdrive conditions from 3-dB to 8-dB gain saturation is discussed. Output RF power degrades significantly in a short period of time. Both transient and permanent degradation of electronic properties of the device are identified. After high RF gain compression levels, DC characteristics like the threshold voltage $\left(V_{T}\right)$ and gate leakage current change permanently. Detailed microscopic changes in the electronic structure of the device were studied by performing simultaneous low-frequency noise measurements of gate and drain currents before and after stress. The channel was found to be immune to the whole stress regime with no increase of the Hooge parameter. On the other hand, activation of unstable defects and then an increase of the defect density near the gate metal semiconductor interface were observed from gate noise measurements. A point defect located at around $4.5 \mathrm{~nm}$ from the gate metal semiconductor interface with activation energy of $0.9 \mathrm{eV}$ below the AIGaN conduction band edge was determined from random telegraph noise measurements. The role of forward gate biasing as a failure mechanism is also discussed.
\end{abstract}

Index Terms-Device reliability, GaN devices, low-frequency noise (LFN), random telegraph switching (RTS) noise.

\section{INTRODUCTION}

A $1 \mathrm{GaN} / \mathrm{GaN}$ transistors have demonstrated exceptional performance for both high-frequency and high-power applications [1], [2]. As device designs are optimized for achieving higher RF power levels, there is an increased need to assess their long-term reliability in actual operating conditions. DC stress tests are typically performed to determine RF reliability, but this approach is often fraught with problems. For instance, some of the failure mechanisms which are active under DC stress may not be the same under RF operation [3]. However, the relative simplicity of the tests renders them useful. A better experiment is the RF operating life time test which is performed to extrapolate RF reliability of the device. However, high temperatures used in the stress accelerate thermally activated processes more than the electric field-driven processes [4]. In this regard, one practical test that can be performed readily and mimics RF failure mechanisms more closely is the RF over-

Manuscript received July 24, 2011; revised September 12, 2011; accepted October 13, 2011. Date of publication October 25, 2011; date of current version March 7, 2012. This work was supported by AFOSR MURI Grant no. FA9550-08-1-0264

The authors are with the Department of Electrical and Computer Engineering, University of Florida, Gainesville, FL 32611 USA (e-mail: phemantrao@ufl.edu).

Color versions of one or more of the figures in this paper are available online at http://ieeexplore.ieee.org.

Digital Object Identifier 10.1109/TDMR.2011.2173497 drive stress. Power amplifiers are typically biased for higher gain saturation to optimize both efficiency and output power [5]. Also, since these devices are biased at the edge of gain compression levels, a slight change (transient or permanent) in the device characteristics can lead to impedance mismatch and thus drive the device into higher gain compression levels or generate spurious device oscillations. This type of stress not only enables a study of RF failure mechanisms but can also give insights into the limits of the device to withstand high RF power levels [6]. Another integral part of studying reliability is to be able to characterize electronic defects both as-built and poststress. Furthermore, one should be able to distinguish between transient effects like mobile charge trapping-detrapping from permanent trap creation and be able to physically locate these phenomena and associated defects. Low-frequency noise (LFN) can play a central role in defect spectroscopy [7]-[9]. Studying gate and channel noise gives spatial locations of these defects giving insights into physical locations of degradation and failure mechanisms [10]. Therefore, this work uses LFN measurements as a reliability characterization tool to systematically probe microscopic degradation in the electronic structure of the gate stack and the channel region under high RF overdrive condition. Although similar studies have been done on GaAs devices, very few studies exist on GaN high electron mobility transistors (HEMTs) [3], [11], [12].

\section{EXPERIMENTAL Details}

\section{A. Device Specifications}

The devices under test are $18 \mathrm{~nm}$ thick $\mathrm{Al}_{0.26} \mathrm{Ga}_{0.74} \mathrm{~N}$ barrier-based GaN transistors grown on silicon substrate. They have a $1.5 \mathrm{~nm}$ thick GaN capping layer which is covered with $\mathrm{Si}_{3} \mathrm{~N}_{4}$ passivation layer on top of the gate-drain and gate-source access regions. The gate length and ten gate finger periphery are $0.5 \mu \mathrm{m}$ and $10 \times 200 \mu \mathrm{m}$, respectively. They have a mushroomshaped gate and a source-connected field plate extension. More details of the device specifications can be found in a previous work [10]. Maximum operating frequency is around $6 \mathrm{GHz}$. The device is fully encapsulated in a ceramic package which protects it from external light-related instabilities.

\section{B. Experimental Setup and Stress Methodology}

A semi-automated experimental setup is developed to perform RF stressing, DC characterization, and LFN measurements (Fig. 1). A circulator is utilized on the RF generator side to measure reflected power from the gate terminal. It 


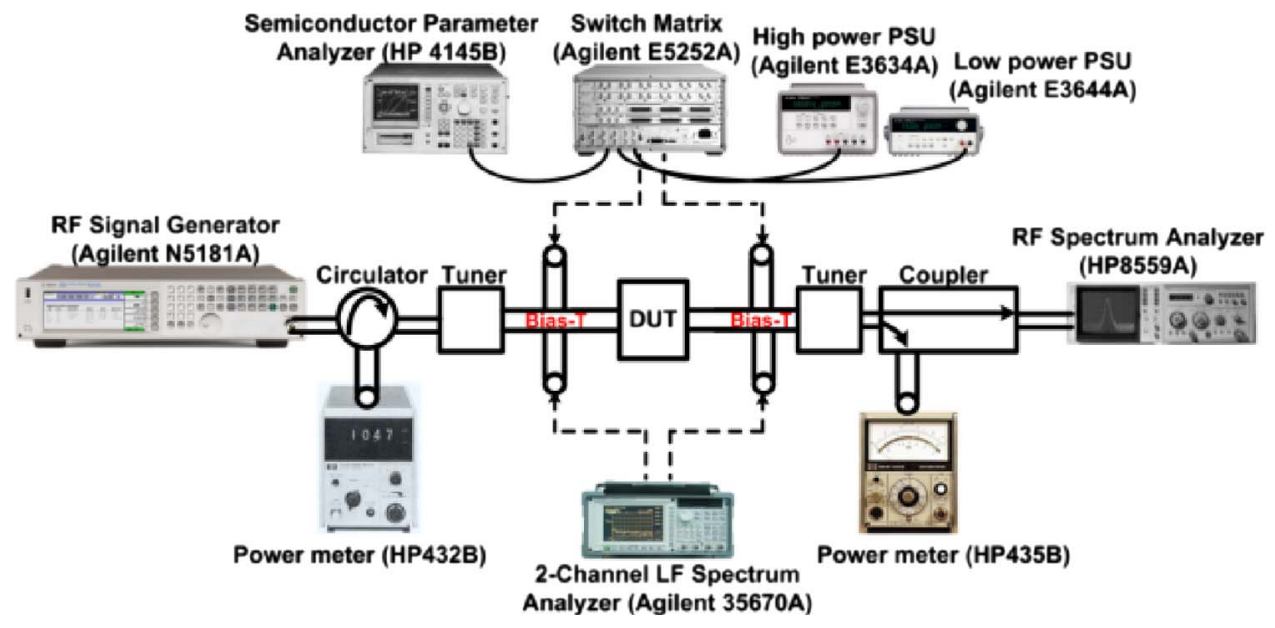

Fig. 1. Experimental setup to perform DC characterization, RF stress, and LFN measurements.

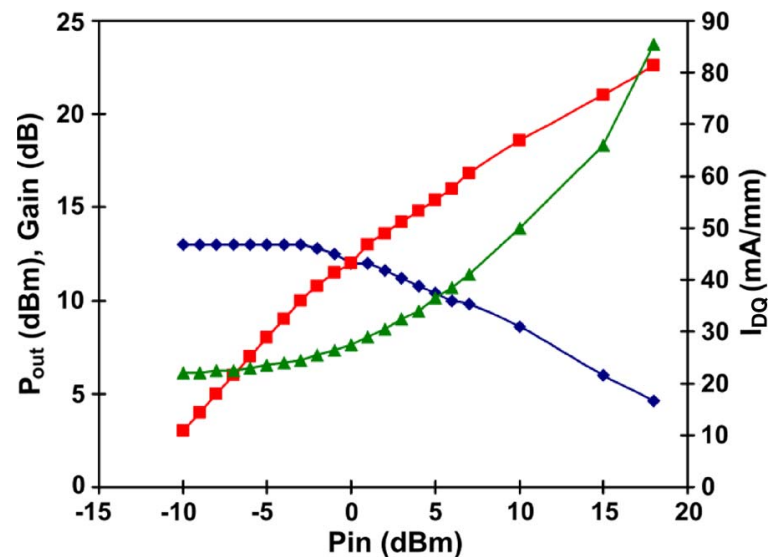

Fig. 2. RF power measurement at $3 \mathrm{GHz}$. Power gain (diamond) and output power (squares) on the left axis. DC quiescent current (triangles) is shown on the right axis. The $3-\mathrm{dB}$ and $8-\mathrm{dB}$ gain compression occurs at an input power of $6 \mathrm{dBm}$ and $18 \mathrm{dBm}$, respectively.

gives the ability to monitor changes in the power gain of the device during stress due to variation in the input impedance due to possible trapping or self-heating effects. An Agilent $35670 \mathrm{~A}$ spectrum analyzer is used to simultaneously measure gate and drain current LFN. The details of the noise setup can be found in a previous work [10]. The complete setup is computer controlled via Agilent VEE.

The device was biased in a class $\mathrm{AB}$ mode of operation at $V_{D S}=28 \mathrm{~V}$ and $I_{D Q}=25 \mathrm{~mA} / \mathrm{mm}$. The static DC power dissipation in the device was significant, with package temperatures reaching $65^{\circ} \mathrm{C}$ during operation. A channel temperature of $100{ }^{\circ} \mathrm{C}$ was determined based on a thermal resistance of $23{ }^{\circ} \mathrm{C} / \mathrm{W}$ which is a reasonable approximation for this device. The device test fixture was kept at the ambient temperature of $25{ }^{\circ} \mathrm{C}$ throughout the stress duration. RF power gain of the device was characterized before stress to determine the $3-\mathrm{dB}$ and 8-dB gain compression points (Fig. 2). A small signal linear gain of $13 \mathrm{~dB}$ was measured at $3 \mathrm{GHz}$. At $3-\mathrm{dB}$ compression, the input power is around $6 \mathrm{dBm}$, and at $8-\mathrm{dB}$ compression, the input power is around $18 \mathrm{dBm}$. The source and load impedances were initially tuned for maximum gain in the linear regime and then kept fixed for the duration of the stress.

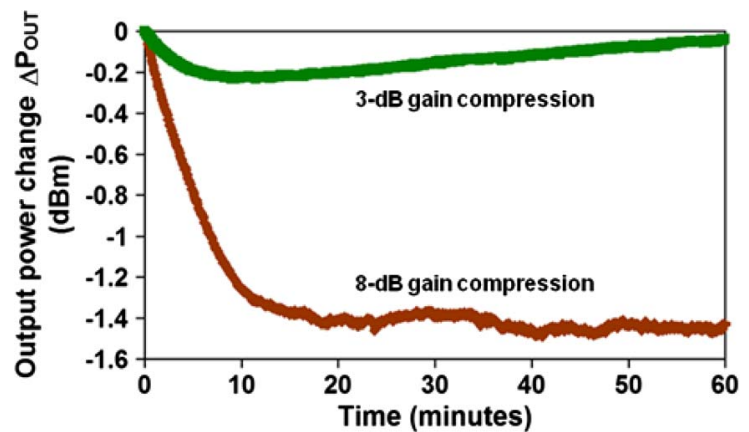

Fig. 3. Degradation of RF output power for 3-dB (green) and 8-dB (brown) gain compression is shown.

RF stress at 3-dB and 8-dB gain compression at $3 \mathrm{GHz}$ was applied for $60 \mathrm{~min}$ (Fig. 3). The device was left to stabilize for few minutes to reach thermal quasi-equilibrium then the input RF power $\left(\mathrm{P}_{\text {IN }}\right)$, output $\mathrm{RF}$ power $\left(\mathrm{P}_{\mathrm{OUT}}\right)$, and quiescent drain current $\left(\mathrm{I}_{\mathrm{DQ}}\right)$ were monitored as a function of time. Before stress application, a complete set of DC and LFN characteristics of the gate and channel were measured. It is important to point out here that RF stress with no gain compression was also performed, and it was found that no degradation of the output RF power $\left(\mathrm{P}_{\text {OUT }}\right)$ occurred. The DC characteristics $\mathrm{V}_{T}, \mathrm{~g}_{m}$, and gate leakage current did not change. Gate and channel noise characteristics were also unchanged. Only higher levels of gain compressions resulted in degradation of device characteristics.

After stress, DC characteristics like threshold voltage, gate leakage current, transconductance, and gate and drain current LFN were measured repeatedly for several weeks to differentiate between temporary and permanent effects.

\section{Results}

Fig. 3 shows the change of RF output power during stress of $1 \mathrm{~h}$ for $3-\mathrm{dB}$ and $8-\mathrm{dB}$ compression.

Despite that these are very short-term stresses, they result in significant changes in power levels. Therefore, a systematic study of degradation can give new insights into these failure mechanisms. 


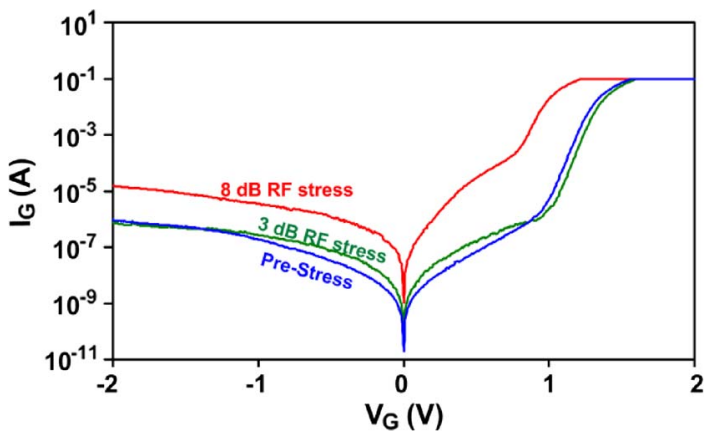

Fig. 4. DC gate $I-V$ for $\mathrm{V}_{\mathrm{DS}}=0$ are shown for prestress (blue), post 3-dB compression stress (green), and post 8-dB compression stress (red).

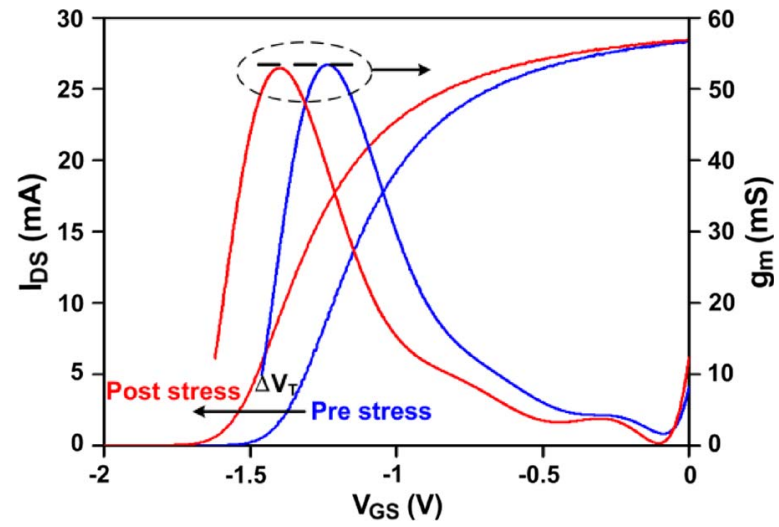

Fig. 5. DC $\mathrm{I}_{D}-\mathrm{V}_{\mathrm{GS}}$ for $\mathrm{V}_{\mathrm{DS}}=80 \mathrm{mV}$ is shown for pre and post 8-dB compression stress.

A significant $(\sim 1.5 \mathrm{~dB})$ power loss takes places in the first $10 \mathrm{~min}$ of stress for high gain compression of $8 \mathrm{~dB}$, and thereafter the power remains relatively steady. On the other hand, a 3-dB gain compression stress causes much less degradation which almost recovers to prestress levels in $60 \mathrm{~min}$. It should be mentioned here that the measured RF power gain followed similar trends since the input power did not change much during stress.

Fig. 4 shows the DC $I-V$ characteristics of the gate before and after stress. DC gate leakage current degraded significantly after stress. In the reverse bias, the gate leakage current increased ten times for $8-\mathrm{dB}$ compression and 2.5 times for $3-\mathrm{dB}$ compression RF stress. Fig. 5 shows the transconductance of the device before and after 8-dB compression stress.

The peak transconductance does not change. However, there is a negative threshold voltage shift which was found to be permanent. After 3-dB compression stress, the channel characteristics (not shown here) remained the same without a change in peak transconductance and threshold voltage.

The microscopic origin of the degradation was studied via gate and channel curent LFN measurements. Fig. 6 shows the evolution of the relative channel noise before and after $8-\mathrm{dB}$ compression stress.

The channel noise goes down after stress. This effect can be explained by the change in the threshold voltage. Noise was measured at a constant gate voltage of $\mathrm{V}_{G}=-1.3 \mathrm{~V}$ and low drain-to-source voltage $\left(\mathrm{V}_{\mathrm{DS}}\right)$ of $80 \mathrm{mV}$. When the threshold voltage becomes more negative, the number of electrons in

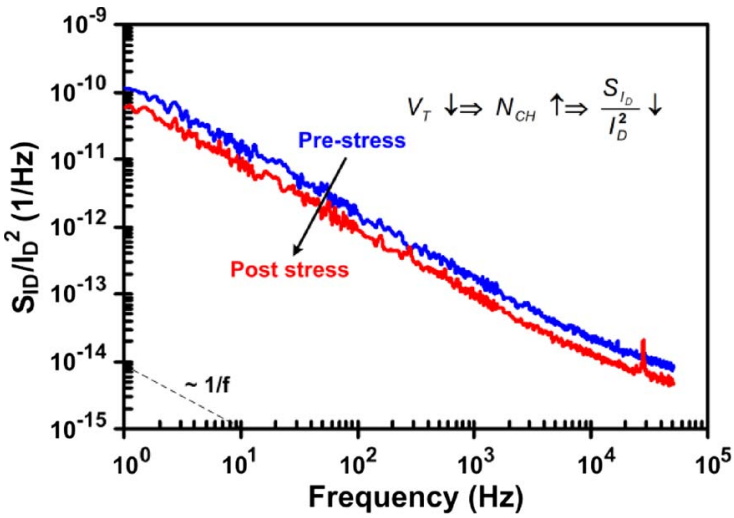

Fig. 6. Relative channel current noise before stress (blue) and after 8-dB compression stress (red).

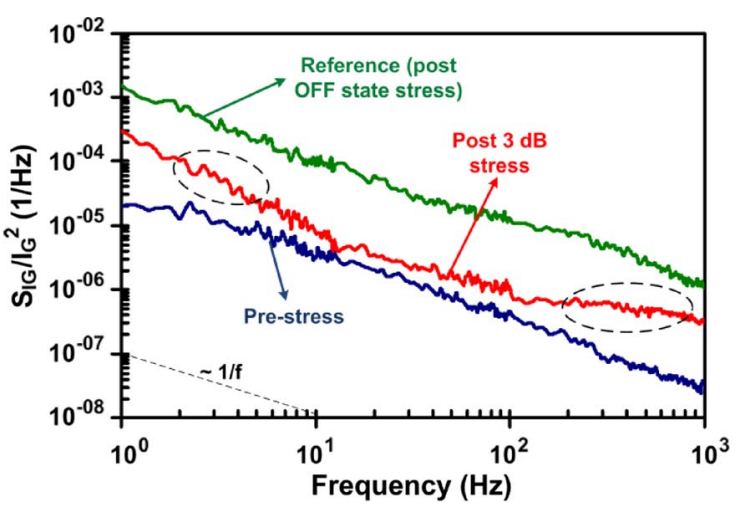

Fig. 7. Relative gate current noise before RF stress (blue), after 3-dB RF stress (red). Noise after DC inverse-piezo electric stress (OFF state) is shown in green. More details of that work can be found in a previous work [19].

the channel at the same gate voltage increases, which leads to a decrease of the relative channel noise magnitude. The quantitative decrease of the noise agrees well with the threshold voltage shift. For the $3-\mathrm{dB}$ compression stress (not shown here), the relative channel noise magnitude remains the same. The Hooge parameter $\left(\alpha_{H}\right)$ was found to be $\sim 10^{-3}$ for both stress conditions. This is also a common value for unstressed $\mathrm{GaN}$ HEMTs [13]. Fig. 7 shows the relative gate noise characteristics of the device after $3-\mathrm{dB}$ compression stress.

It can be seen that post $3-\mathrm{dB}$ compression stress, the gate $1 / \mathrm{f}$ noise increases slightly but also induces temporally unstable Lorentzians indicated in the figure by the dashed ellipsoids. These Lorentzians sometimes also manifested as random telegraph switching (RTS) noise in the time domain. RTS noise is a powerful tool to study single defects [14], [15].

During one of the measurements, a stable RTS was found which lasted for several hours before it disappeared. RTS noise measurements were performed as a function of gate voltage to extract mean up and down time constants from the Poissonian statistics given by

$$
t_{\text {up } / \text { down }} \sim \exp \left(-\frac{t}{\tau_{\text {up } / \text { down }}}\right)
$$

where $\tau_{\text {up/down }}$ is the mean up/down time constant. Fig. 8 shows the extracted up and down time constants as a function of gate voltage. It can be seen that one of these time constants 


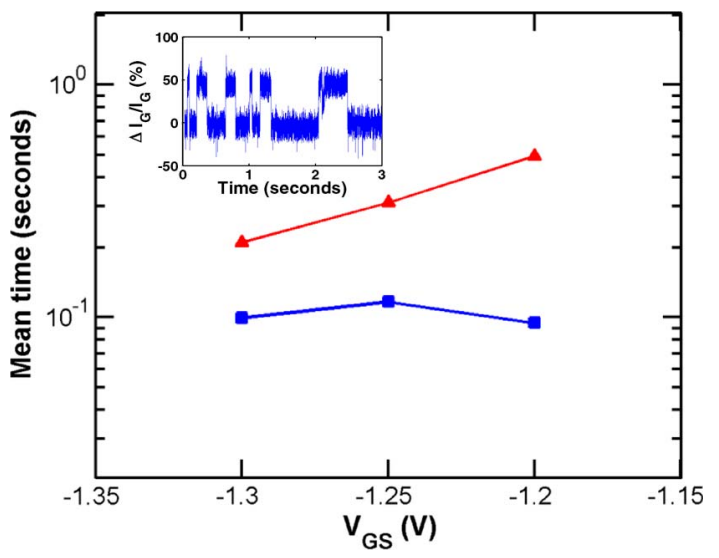

Fig. 8. Mean capture (triangle) and emission times (square) determined from RTS noise data as a function of gate voltage (above threshold voltage). Inset shows the relative gate current fluctuation in the time domain.

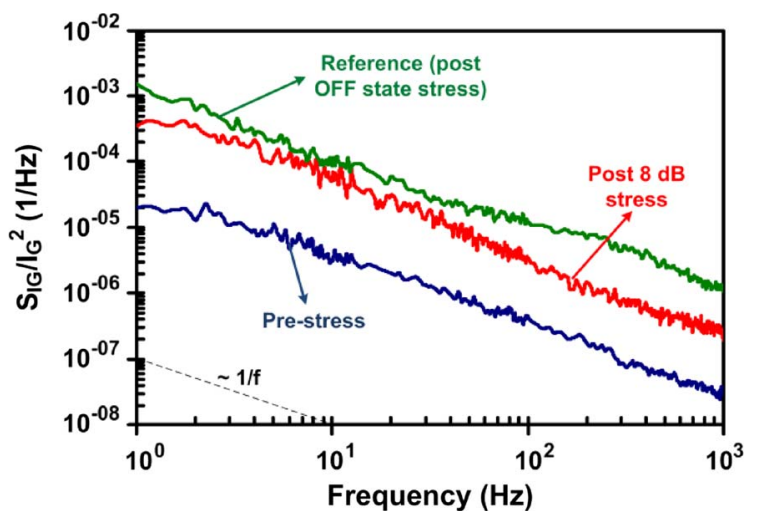

Fig. 9. Relative gate current noise before stress (blue) after 8-dB RF stress (red). Noise after inverse-piezo electric stress (OFF state) is shown in green. More details of that work can be found in a previous work [19].

labeled as the capture time is exponentially dependent on the gate voltage, whereas the other time constant is not. The details of the mechanism are explained in the next section where the trap characteristics are determined by analyzing this data. Fig. 9 shows the gate noise characteristics before and after 8-dB compression stress. It can be observed that the $1 / \mathrm{f}$ noise increases drastically after stress. Furthermore, no distinct Lorentzian components are visible in the spectra after 8-dB compression stress unlike the $3-\mathrm{dB}$ compression stress which resulted in temporally unstable Lorentzians.

\section{Discussion AND Model}

\section{A. Channel Noise Analysis}

It is clear from the channel noise characteristics that microscopic degradation does not occur in the channel region of the device. After the 3-dB compression stress, there is no net change in the relative channel noise characteristics, and its $1 / \mathrm{f}^{\gamma}$-type characteristic is intact. However, for 8 -dB compression stress, a net decrease of channel noise occurs which was found to be permanent. This was explained by the threshold voltage shift which became more negative after stress as pointed out earlier. The Hooge parameter $\left(\alpha_{H}\right)$ remained unchanged

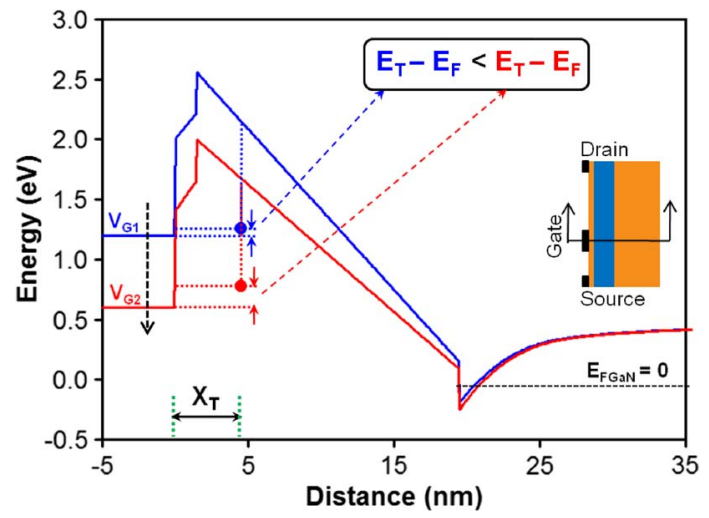

Fig. 10. Conduction band diagram under the gate for two increasing (black dashed arrow) gate voltages $\mathrm{V}_{G 1}$ and $\mathrm{V}_{G 2}$ (above the threshold voltage).

after both stresses confirming the immunity of channel region to the applied RF stress at the microscopic level.

\section{B. Gate Noise Analysis}

Gate leakage current, gate noise, and relative gate noise increased after 8-dB compression stress. 1/f noise is typically linked to interface trap states close to the gate metal/semiconductor interface [11], [16]. A permanent increase of $1 / \mathrm{f}$ noise indicates that trap density near this interface increased, and new defect states were generated during stress. The exact mechanism of DC gate leakage current in GaN HEMTs is still unclear. There is however a consensus that trap states in the AlGaN barrier are responsible for high leakage currents [17], [18]. Therefore, one can conclude that the trap density indeed increased at the interface which explains the gate noise increase. One more interesting aspect of the study was the activation of temporally unstable Lorentzians which gave RTS noise in the time domain after 3-dB compression stress [Fig. 8]. It is known that a two-level switching RTS is a single electron event with a single defect participating in the process [14].

The large observed relative gate current fluctuations point to a localized nature of gate leakage current. Hence, instead of all the ten fingers equally participating in the DC gate leakage current, only one or two fingers participate. Furthermore, one can extract the physical location of this defect along the gateto-channel direction by studying its gate bias dependence. For a single defect, the principle of detailed balance requires

$$
\frac{\bar{\tau}_{c}}{\bar{\tau}_{e}}=g \exp \left(\frac{E_{T}-E_{F}}{k_{B} T}\right)
$$

where $\mathrm{E}_{T}$ is the trap energy level, $\mathrm{E}_{F}$ is the Fermi energy level, $\mathrm{k}_{\mathrm{B}}$ is the Boltzmann constant, $\mathrm{g}$ is the trap degeneracy, and $\mathrm{T}$ is the temperature. Fig. 10 shows the 1-D energy conduction band diagram along gate-to-GaN buffer simulated using a self-consistent Poisson-Schrodinger solver [21]. Analytically, $\mathrm{E}_{T}-\mathrm{E}_{F}$ can be evaluated from the band diagram

$$
\begin{aligned}
E_{T}-E_{F}=q \Phi_{B} & +x_{1}\left(k_{1} V_{G}+E_{\mathrm{PGaN}}\right)+\Delta E_{C} \\
& -x_{T}\left(k_{2} V_{G}+E_{\mathrm{PAlGaN}}\right)-\left(E_{C}-E_{T}\right)
\end{aligned}
$$

where $\Phi_{B}$ is the metal/semiconductor Schottky barrier height, $\mathrm{k}_{1} \mathrm{~V}_{G}$ is the electric field component in the GaN capping layer 


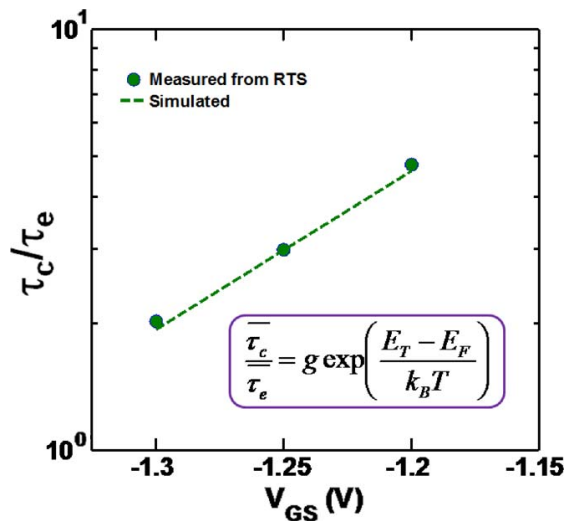

Fig. 11. Ratio of mean time switching constants (dots) is plotted as a function of gate voltage. The dashed line shows the simulated value of this ratio equation relation (2) and (3).

resulting from applying $\mathrm{V}_{G}, \mathrm{E}_{\mathrm{PGaN}}$ is the electric field in the GaN capping layer due to spontaneous polarization, $\Delta \mathrm{E}_{C}$ is the conduction band discontinuity between GaN capping and AlGaN barrier, $\mathrm{x}_{T}$ is the distance of the trap location along the gate-to-GaN buffer direction from the GaN capping/AlGaN barrier interface, $\mathrm{k}_{2} \mathrm{~V}_{G}$ is the electric field component in the $\mathrm{AlGaN}$ barrier layer resulting from applying $\mathrm{V}_{G}$, and $\mathrm{E}_{\mathrm{PAlGaN}}$ is the electric field in the $\mathrm{AlGaN}$ barrier layer due to net spontaneous and piezoelectric polarization. Assuming $g=1$ and differentiating eq. (3) with respect to the gate voltage

$$
\frac{\partial\left(E_{T}-E_{F}\right)}{\partial V_{G}}=k_{B} T \frac{\partial \ln \left(\frac{\bar{\tau}_{C}}{\bar{\tau}_{E}}\right)}{\partial V_{G}}=k_{1} x_{1}-x_{T} k_{2} \cong-x_{T} k_{2}
$$

is the slope which gives the location of the defect $\left(\mathrm{x}_{T}\right)$, and the intercept of (3) gives the energy of the defect $\left(\mathrm{E}_{C}-\mathrm{E}_{T}\right)$ if the values of $\mathrm{k}_{1}, \mathrm{k}_{2}, \Phi_{B}, \Delta \mathrm{E}_{C}, \mathrm{E}_{\mathrm{PGaN}}, \mathrm{E}_{\mathrm{PAlGaN}}$ are known. Fig. 11 shows the measured RTS data and simulated $\tau_{C} / \tau_{E}$ from the band diagram.

An excellent agreement between the measured and simulated values is obtained for a trap located $4.5 \mathrm{~nm}$ from the $\mathrm{metal} / \mathrm{semiconductor} \mathrm{interface} \mathrm{and} 0.9 \mathrm{eV}$ below the conduction band edge of the AlGaN barrier. A Schottky barrier height $\left(\varphi_{B}\right)$ of $0.8 \mathrm{eV}$ is assumed. It has been postulated in the literature that point defects aggregate around dislocations which act as stepping stones for defect-assisted tunnelling [17]. Also, these defects are located close to the Fermi level making them accessible from the gate terminal. It can be argued that the overall increase of the trap density in the gate stack observed at high RF stress (8-dB compression) is a result of an overall increase of these individual point defects. However, more work is required to confirm this hypothesis possibly based on currentand temperature-driven defect diffusion mechanisms.

\section{Failure Mechanism}

Another issue which needs further discussion is the physical mechanism for the observed degradation. To answer this question, it is appropriate to discuss the understanding gained from the systematic DC stress measurements. The authors have

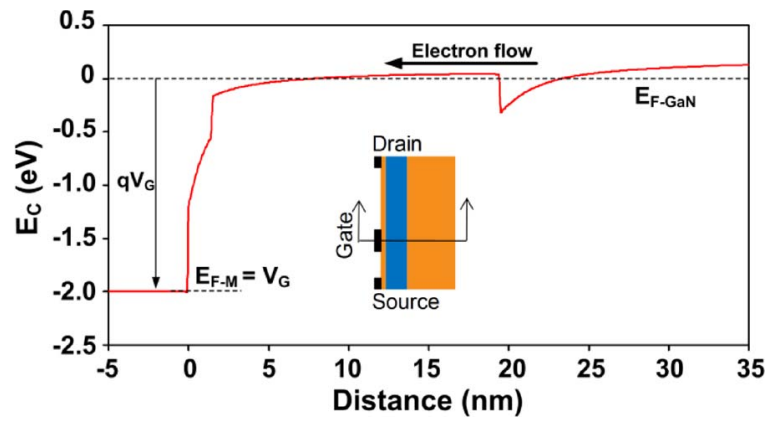

Fig. 12. Conduction band diagram under the gate for $\mathrm{V}_{\mathrm{GS}}=2 \mathrm{~V}$. Most of the voltage drop occurs very near to the gate metal/GaN capping semiconductor interface and GaN capping layer. The dashed line shows the Fermi level in the GaN channel layer. The arrow shows the direction of electron motion for forward gate current.

performed DC stress for a range of gate and drain biases and characterized permanent and temporary degradation by noise measurements [19]. The stress regime where large negative DC gate voltage is applied on the device with source and drain grounded, degradation of gate $1 / \mathrm{f}$ noise was observed with no channel degradation. This signature is similar to what is observed in this experiment, and the failure mechanism is linked to inverse piezo-electric stress.

However, the device also experiences elevated temperatures due to static and dynamic current in the channel. It has been shown in the literature that thermo-elastic stress counteracts the effect of inverse-piezo electric stress, thereby increasing the robustness of the gate stack at higher temperatures [20].

This brings attention to a failure mechanism which is particularly relevant for high RF overdrive conditions. During large RF positive voltage swings on the gate, high forward currents flow through the gate Schottky junction. Fig. 12 shows the simulated 1-D conduction band diagram at a positive gate voltage of $2 \mathrm{~V}$ along the gate to $\mathrm{GaN}$ buffer layer direction without solving the transport equations. Its clear from the diagram that most of the potential drop takes place very near to the metal/semiconductor interface and $\mathrm{GaN}$ capping layer due to large opposite built-in piezoelectric polarization electric field in the AlGaN barrier. Thus, carriers which are injected from the 2-DEG channel dissipate most of their energy into the gate metal/semiconductor junction and GaN capping layer, thereby the probability of degradation increases in this region. This agrees well with the gate noise characteristics which confirmed degradation right under the gate metal. To further corroborate this, the authors performed a forward gate DC voltage stress. Fig. 13 shows the results of DC forward voltage stress on the gate diode characteristics with source and drain grounded. It can be seen that gate leakage current degrades irreversibly after $\mathrm{V}_{G}=1 \mathrm{~V}$ DC stress. The threshold voltage (not shown here) also shifted toward more negative values. These two effects are well correlated to the DC degradation effects observed after the 8-dB compression RF stress. Furthermore, noise measurements were also carried out on DC stressed devices which agreed well with results from RF stress, thus, confirming the role of forward gate bias as a primary failure mechanism in this study. 


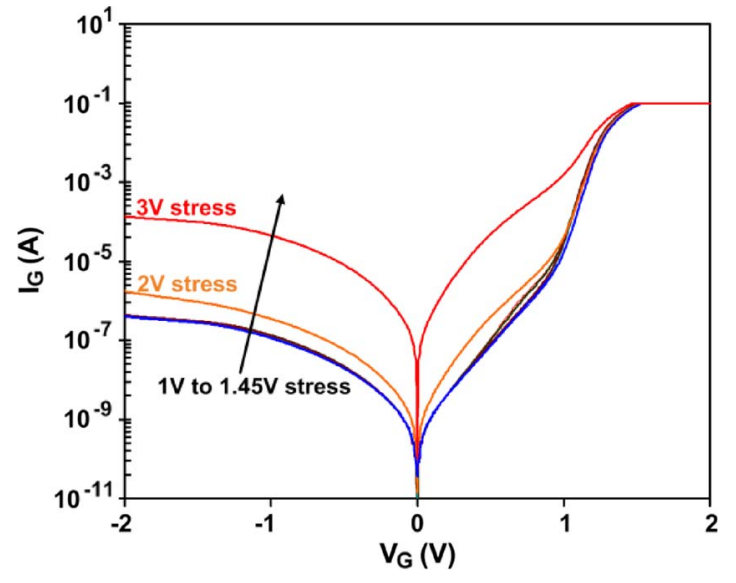

Fig. 13. Gate diode $I-V$ characteristics for increasing (arrow direction) forward DC gate voltage stress from $1 \mathrm{~V}$ to $3 \mathrm{~V}$ of 5 min each.

\section{CONCLUSION}

In summary, the effect of short-term high-power RF stress on $\mathrm{AlGaN} / \mathrm{GaN}$ HEMTs is characterized. It was found that degradation occurs right under the gate in the gate metal-semiconductor interface and GaN capping layer without affecting the channel. Gate noise measurements pointed out that an increase of trap density near the metal-semiconductor interface occurred after high gain compression RF stress. Individual point defects which were activated after low RF power stress were also characterized. The role of forward gate biasing as a failure mechanism was discussed. This study also points out that when the reliability of the gate stack at high RF compression levels is considered, a simpler gate forward biased DC stress test can replace the high gain compression RF stress measurment. Finally, it was shown that LFN is an insightful defect spectroscopic tool to study electronic device reliability.

\section{REFERENCES}

[1] Y.-F. Wu, M. Moore, A. Saxler, T. Wisleder, and P. Parikh, "40-W/mm double field-plated GaN HEMTs," in Proc. 64th Device Res. Conf., 2006, pp. 151-152.

[2] J. W. Chung, W. E. Hoke, E. M. Chumbes, and T. Palacios, "AlGaN/GaN HEMT with 300-GHz f_\{max $\}$," IEEE Electron Device Lett., vol. 31, no. 3, pp. 195-197, Mar. 2010.

[3] J. Joh and J. A. del Alamo, "RF power degradation of GaN high electron mobility transistors," in IEDM Tech. Dig., 2010, pp. 20.2.1-20.2.4.

[4] A. M. Conway, M. Chen, P. Hashimoto, P. J. Willadsen, and M. Micovic, "Accelerated RF life testing of Gan Hfets," in Proc. 45th Annu. IEEE Int. Reliab. Phys. Symp., 2007, pp. 472-475.

[5] H. Hasegawa, K. Katsukawa, T. Itoh, T. Noguchi, and Y. Kaneko, "High reliability power GaAs MESFET under RF overdrive condition," in Proc. IEEE MTT-S Int. Microw. Symp. Dig., 1993, vol. 1, pp. 289-292.

[6] M. Borgarino, R. Menozzi, D. Dieci, L. Cattani, and F. Fantini, "Reliability physics of compound semiconductor transistors for microwave applications," Microelectron. Reliab., vol. 41, no. 1, pp. 21-30, Jan. 2001.

[7] M. M. Jevtic, "Noise as a diagnostic and prediction tool in reliability physics," Microelectron. Reliab., vol. 35, no. 3, pp. 609-612, Mar. 1995.

[8] L. K. J. Vandamme, "Noise as a diagnostic tool for quality and reliability of electronic devices," IEEE Trans. Electron Devices, vol. 41, no. 11, pp. 2176-2187, Nov. 1994.

[9] E. Simoen and C. Claeys, "Reliability aspects of the low-frequency noise behaviour of submicron CMOS technologies," Semicond. Sci. Technol., vol. 14, no. 8, pp. R61-R71, Aug. 1999.

[10] H. Rao and G. Bosman, "Simultaneous low-frequency noise characterization of gate and drain currents in $\mathrm{AlGaN} / \mathrm{GaN}$ high electron mobility transistors," J. Appl. Phys., vol. 106, no. 10, pp. 103712-1-103712-5, Nov. 2009.
[11] B. Lambert, N. Malbert, F. Verdier, N. Labat, A. Touboul, and L. K. J. Vandamme, "Low frequency gate noise in a diode-connected MESFET: Measurements and modeling," IEEE Trans. Electron Devices, vol. 48, no. 4, pp. 628-633, Apr. 2001.

[12] N. Saysset-Malbert, B. Lambert, C. Maneux, N. Labat, A. Touboul, Y. Danto, P. Huguet, P. Auxemery, and F. Garat, "Investigation on GaAs power MESFETs submitted to RF life-test by LF noise and drain current transient analysis," in Proc. GaAs Reliab. Workshop, 1999, pp. 77-83.

[13] S. Rumyantsev, M. E. Levinshtein, R. Gaska, M. S. Shur, J. W. Yang, and M. A. Khan, "Low-frequency noise in AlGaN/GaN heterojunction field effect transistors on $\mathrm{SiC}$ and sapphire substrates," J. Appl. Phys., vol. 87, no. 4, pp. 1849-1854, Feb. 2000.

[14] M. J. Kirton and M. J. Uren, "Noise in solid-state microstructures: A new perspective on individual defects, interface states and low-frequency $(1 / f)$ noise," Adv. Phys., vol. 38, no. 4, pp. 367-468, Nov. 1989.

[15] N. V. Amarasinghe, Z. Çelik-Butler, and A. Keshavarz, "Extraction of oxide trap properties using temperature dependence of random telegraph signals in submicron metal-oxide-semiconductor field-effect transistors," J. Appl. Phys., vol. 89, no. 10, pp. 5526-5532, May 2001.

[16] H. Rao and G. Bosman, "Device reliability study of high gate electric field effects in $\mathrm{AlGaN} / \mathrm{GaN}$ high electron mobility transistors using low frequency noise spectroscopy," J. Appl. Phys., vol. 108, no. 5, pp. 053707 1-053707-5, Sep. 2010.

[17] H. Zhang, E. J. Miller, and E. T. Yu, "Analysis of leakage current mechanisms in Schottky contacts to $\mathrm{GaN}$ and $\mathrm{Al}_{0.25} \mathrm{Ga}_{0.75} \mathrm{~N} / \mathrm{GaN}$ grown by molecular-beam epitaxy," J. Appl. Phys., vol. 99, no. 2, pp. 023 703-1023 703-6, Jan. 2006.

[18] S. Karmalkar, D. M. Sathaiya, and M. S. Shur, "Mechanism of the reverse gate leakage in AlGaN/GaN high electron mobility transistors," Appl. Phys. Lett., vol. 82, no. 22, pp. 3976-3978, Jun. 2003.

[19] H. Rao and G. Bosman, "Device reliability study of AlGaN/GaN high electron mobility transistors under high gate and channel electric fields via low frequency noise spectroscopy," Microelectron. Reliab., vol. 50, no. 9-11, pp. 1528-1531, Sep. 2010.

[20] T. Beechem, A. Christensen, D. S. Green, and S. Graham, "Assessment of stress contributions in GaN high electron mobility transistors of differing substrates using Raman spectroscopy," J. Appl. Phys., vol. 106, no. 11, pp. 114 509-1-114 509-9, Dec. 2009.

[21] S. Birner, T. Zibold, T. Andlauer, T. Kubis, M. Sabathil, A. Trellakis, and P. Vogl, "nextnano: General purpose 3-D simulations," IEEE Trans. Electron Devices, vol. 54, no. 9, pp. 2137-2142, Sep. 2007.

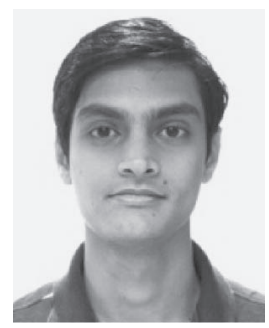

Hemant P. Rao was born in New Delhi, India, in 1985. He received the B.Tech. degree in electronics and communication engineering from National Institute of Technology, Bhopal, India, in 2007, the M.S degree in electrical and computer engineering from the University of Florida, Gainesville, in 2008 where he is currently working toward the Ph.D. degree in electrical and computer engineering.

He interned with the Phase Change Memory group at Intel Corporation, Santa Clara, CA, in 2011, and the SPICE modeling group at National Semiconductor Corporation, Santa Clara, CA, in 2010. His research interests include low-frequency noise characterization, semiconductor device reliability, and modeling.

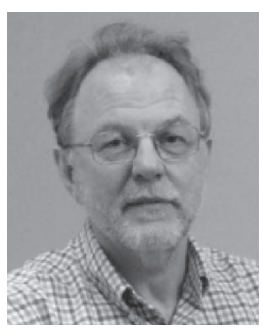

Gijs Bosman (M'82-SM'89) received the B.S., M.S., and Ph.D. degrees in physics from the University of Utrecht, Utrecht, The Netherlands in 1971, 1976, and 1981, respectively.

In 1981, he joined the Department of Electrical and Computer Engineering, University of Florida, Gainesville, where he is currently a Professor. He has published more than 100 refereed journal and conference papers on these topics. His research interests include the electrical noise and charge transport properties of semiconductor devices and circuits.

Dr. Bosman is a member of the American and Dutch Physical Societies, and a senior member of the Institute of Electrical and Electronics Engineers. In addition to the College of Engineering Teacher of the Year Award for 1998-1999, he received several university and departmental awards for teaching and research. 